\title{
BMJ Open Data quality and 30-day survival for out- of-hospital cardiac arrest in the UK out- of-hospital cardiac arrest registry: a data linkage study
}

\author{
Sangeerthana Rajagopal, ${ }^{1,2}$ Scott J Booth, ${ }^{1}$ Terry P Brown, ${ }^{1}$ Chen Ji, ${ }^{1}$ \\ Claire Hawkes, ${ }^{1}$ A Niroshan Siriwardena, ${ }^{3}$ Kim Kirby, ${ }^{4}$ Sarah Black, ${ }^{4}$ \\ Robert Spaight, ${ }^{5}$ Imogen Gunson, ${ }^{6}$ Samantha J Brace-McDonnell,, ${ }^{1,2}$ \\ Gavin D Perkins, ${ }^{1,2}$ on behalf of OHCAO collaborators
}

To cite: Rajagopal S, Booth SJ, Brown TP, et al. Data quality and 30-day survival for outof-hospital cardiac arrest in the UK out-of-hospital cardiac arrest registry: a data linkage study. BMJ Open 2017;7:e017784. doi:10.1136/ bmjopen-2017-017784

- Prepublication history and additional material for this paper are available online. To view these files, please visit the journal online (http://dx.doi. org/10.1136/bmjopen-2017017784).

Received 16 May 2017 Revised 7 September 2017 Accepted 14 September 2017

\section{CrossMark}

${ }^{1}$ Warwick Clinical Trials Unit, Warwick Medical School, University of Warwick, Coventry, UK

${ }^{2}$ Heart of England NHS Foundation Trust, Birmingham, UK

${ }^{3}$ School of Health and Social Care, University of Lincoln, Lincoln, UK

${ }^{4}$ South Western Ambulance Service NHS Foundation Trust, Exeter, UK

${ }^{5}$ East Midlands Ambulance Service NHS Trust, Nottingham, UK

${ }^{6}$ West Midlands Ambulance Service NHS Foundation Trust, Brierley Hill, UK

Correspondence to

Professor Gavin D Perkins; g.d.perkins@warwick.ac.uk

\section{ABSTRACT}

Objectives The Out-of-Hospital Cardiac Arrest Outcomes (OHCAO) project aims to understand the epidemiology and outcomes of out-of-hospital cardiac arrest (OHCA) across the UK. This data linkage study is a subproject of OHCAO. The aim was to establish the feasibility of linking OHCAO data to National Health Service (NHS) patient demographic data and Office for National Statistics (ONS) date of death data held on the NHS Personal Demographics Service (PDS) database to improve OHCAO demographic data quality and enable analysis of 30-day survival from OHCA. Design and setting Data were collected from 1 January 2014 to 31 December 2014 as part of a prospective, observational study of OHCA attended by 10 English NHS Ambulance Services. 28729 OHCA cases had resuscitation attempted by Emergency Medical Services and were included in the study. Data linkage was carried out using a data linkage service provided by NHS Digital, a national provider of health-related data. To assess data linkage feasibility a random sample of 3120 cases was selected. The sample was securely transferred to NHS Digital to be matched using OHCAO patient demographic data to return previously missing demographic data and provide ONS date of death data.

Results A total of 2513 (80.5\%) OHCAO cases were matched to patients in the NHS PDS database. Using the linkage process, missing demographic data were retrieved for $1636(72.7 \%)$ out of 2249 OHCAO cases that had previously incomplete demographic data. Returned ONS date of death data allowed analysis of 30-day survival status. The results showed a 30 -day survival rate of $9.3 \%$, reducing unknown survival status from $46.1 \%$ to $8.5 \%$. Conclusions In this sample, data linkage between the OHCAO registry and NHS PDS database was shown to be feasible, improving demographic data quality and allowing analysis of 30-day survival status.

\section{INTRODUCTION}

Every year in the UK there are around 60000 out-of-hospital cardiac arrests (OHCAs) attended by Emergency Medical Services (EMS) of which approximately 28000 have

\section{Strengths and limitations of this study}

- Data points collected as part of the Out-of-Hospital Cardiac Arrest Outcomes (OHCAO) project were based on established Utstein guidelines.

- The quality of demographic data collected by the OHCAO project was first improved through a list cleaning and patient status service provided by NHS Digital.

- Following list cleaning, exact data matches with Office for National Statistics date of death data allowed calculation of 30-day survival status.

- Provision of National Health Service numbers from OHCAO and NHS Digital provides potential for following long-term survival outcomes in OHCA patients through data linkage.

- Improved data linkage is reliant on improved data capture of patient demographic data by ambulance services.

resuscitation attempted. ${ }^{12}$ This group suffers significant mortality and morbidity, ${ }^{3}{ }^{4}$ and improving outcomes from OHCA remains a worldwide research priority. ${ }^{5}$

Collecting high-quality data is essential as this forms the basis of decisions that ultimately impact on changes in care and healthcare resource allocation. Since 2011, survival to hospital discharge rates for OHCA has been reported as part of the National Health Service (NHS) England Ambulance Quality Indicators (AQIs), with significant variation reported ranging from $2.2 \%$ to $12.0 \% .^{6}$ Regional variation in survival rates has also been observed worldwide. ${ }^{7-9}$ Lilford et al highlighted that an important source of variation in reporting outcomes can be traced to the quality of data that results are based on. ${ }^{10}$ Collecting survival to discharge data in England is a challenging process for 
ambulance services as it involves tracking the patient's survival status directly with hospital emergency departments, which is time consuming and can be hindered by governance issues. ${ }^{11} 12$

Data collected in international OHCA registries enable comparisons of OHCA epidemiology and outcomes across different EMS systems. ${ }^{13-15}$ The Utstein guidelines provide a structured template for collecting data on OHCA processes to support such comparisons. ${ }^{16}$ To facilitate ease of reporting, the updated Utstein guidelines recommend collecting either 30-day survival or survival to hospital discharge as a core outcome. ${ }^{17}$ The research literature suggests most international registries are able to report either of these OHCA outcome measures. ${ }^{13-15}$ A recent example is the EuReCa ONE study which aimed to benchmark OHCA incidence, process and outcomes across 27 European countries and reported a combined survival to discharge or 30-day survival rate which ranged between $1.1 \%$ and $30.8 \% .^{15}$

Data linkage methodology has increasingly been used in medical research to establish outcomes. It involves linking information together from different sources that belong to the same individual. ${ }^{18}$ Data linkage has been used by regional and national OHCA databases to confirm survival status through linkage with mortality databases. ${ }^{41920}$ Data linkage can address missing data issues, providing a centralised, high-quality database for research and service appraisal with the potential to allow longitudinal surveillance of OHCA patients.

The Out-of-Hospital Cardiac Arrest Outcomes (OHCAO) project is funded by the Resuscitation Council (UK), British Heart Foundation, and managed by the University of Warwick. It is a prospective observational study investigating the epidemiology and outcomes of OHCA patients across the UK. ${ }^{21}{ }^{22}$ This paper presents a subproject of the OHCAO project aiming to establish the feasibility of linking OHCAO registry data to NHS patient demographic data and Office for National Statistics (ONS) mortality data through the NHS Digital list cleaning and patient status service.

\section{METHODS \\ Setting}

The OHCAO project established a national UK OHCA registry to collect process and outcome data to facilitate OHCA research and quality improvement. Detailed information about the OHCAO project is available in the study protocol. ${ }^{21}$ The 10 English NHS ambulance services collecting data for the OHCAO project cover approximately 54 million people, equating to $99.7 \%$ of the England population and $83.9 \%$ of the UK population. ${ }^{23}$ Data were collected from 1 January 2014 to 31 December 2014 on 28729 patients suffering OHCA in whom resuscitation was attempted by statutory EMS (an incidence rate of 53.2 per 100000 of the English population). ${ }^{22}$ This figure was reached after excluding individuals who achieved return of spontaneous circulation (ROSC) before arrival of EMS $(n=1711)$ and where resuscitation was not attempted as per national guidelines ${ }^{24}$ due to the presence of a do not attempt resuscitation order $(n=387)$ or signs incompatible with life or where resuscitation attempts would be futile $(n=5403)$.

\section{Aims and objectives}

The overall aim of this project was to investigate the feasibility of linking a sample of OHCAO 2014 data to NHS patient demographic data and ONS date of death data held on the NHS Personal Demographics Service (PDS) database, using the NHS Digital list cleaning and patient status service, to improve OHCAO demographic data quality and allow calculation of 30-day survival from OHCA. The objectives were to (1) assess the match rate of combinations of OHCAO patient demographic variables in the sample (NHS number, surname, forename, date of birth (DOB) and home postcode) for linking to the NHS PDS database through NHS Digital list cleaning; (2) assess improvements in the completeness of OHCAO patient demographic variables through NHS Digital list cleaning and (3) create a linked OHCAO and NHS PDS database allowing analysis of 30-day survival from OHCA.

\section{OHCAO project data collection}

Core and supplemental Utstein variables were collected encompassing demographic, system, process and outcome data. ${ }^{16}$ Each ambulance service has its own methods for OHCA case ascertainment, for example, electronic searches of patient report form databases for diagnostic codes indicating cardiac arrest. A trained member of the ambulance service clinical audit team entered eligible cases into a cardiac arrest database, followed by data cleaning and verification processes. Survival to hospital discharge data was collected directly from hospitals by the clinical audit team if data-sharing protocols were in place. Each ambulance service uploaded its data via a secure server to the OHCAO registry which is stored at the University of Warwick.

\section{OHCAO data sample}

To assess feasibility while minimising costs associated with data linkage, the analysis presented here represents a $10.9 \%$ sample of the 2014 OHCAO data, comprising 3120 OHCA patients. To avoid selection bias, the sample was selected using simple random sampling and stratified by ambulance service.

\section{OHCAO data linkage to ONS mortality data}

OHCAO to NHS PDS data linkage approval was received after submitting an application to the NHS Digital Data Access Request Service; additional approval was obtained from ONS for the release of mortality data. OHCAO submitted 3120 cases to NHS Digital, via the NHS Digital secure transfer system, detailing the following patient demographic variables of varying completeness: NHS number, surname, forename, DOB and home postcode.

NHS Digital is the national provider of data relating to health and social care in England. OHCAO used 
the NHS Digital list cleaning and patient status service. The list cleaning service was used to validate submitted demographic data to ensure accuracy and improve data linkage outcomes. Validation was achieved by NHS Digital matching submitted demographic variables to NHS patient demographic data held on the PDS database. The PDS database is a national electronic database containing NHS patient demographic information, including NHS number, name and address. For each matched case, NHS Digital was asked to provide OHCAO with the following patient demographic information: NHS number, surname, forename and home postcode. These data were used to improve the percentage of missing data for these variables in the OHCAO sample.

NHS Digital used both automatic and manual matching techniques, using a combination of deterministic and probabilistic data linkage methods. ${ }^{25}{ }^{26}$ In deterministic data linkage, it is decided a priori what combination of patient identifiers to match on (eg, NHS number and DOB) and only complete agreement between records is considered a match. In probabilistic data linkage, weights are assigned to different patient identifiers (based on their discriminatory power) to assess the probability that two records are a match. ${ }^{27}$ Cases were initially submitted for automatic matching which used a decision tree algorithm to provide matches. A subset of cases that failed automatic matching were resubmitted for manual matching.

As part of the patient status service, NHS Digital was also able to provide a date of death for deceased patients. The date of death data was held in the NHS PDS database and was sourced from ONS mortality data. OHCAO required information on deaths from 1 January 2014 until 31 January 2015. This was used to calculate 30-day survival. Where no date of death was provided, the patient was categorised as alive.

\section{ONS date of death data}

ONS mortality data contain all deaths registered in England and Wales. When a person dies, a formal medical certificate of death is produced, usually by a doctor, and which includes date of death. There is then a legal requirement for the death to be registered with the Registrar of Births, Deaths and Marriages through the local register office. The registration is typically performed by a close relative. The certification, and subsequent registration, of death may be delayed if the death is referred to a coroner for investigation (eg, if cause of death is unknown). However, the majority of deaths in England and Wales are registered within 5 days of the death date. ${ }^{28}{ }^{29}$ ONS receives death data in electronic form directly from register offices. All data received are subject to both initial and routine data quality and validation processes and are collected in line with the Statistics and Registration Service Act 2007. ${ }^{28}$

\section{Analysis}

An analysis was conducted to assess how particular demographic data points enabled linkage with NHS PDS data and whether data linkage improved the completeness of patient demographic data. This was done descriptively with breakdowns of data linkage match rates for all combinations of the OHCAO demographic variables sent to NHS Digital for data linkage.

The combined linked dataset was analysed to investigate 30-day survival rates calculated by evaluating if patients were alive $\geq 30$ calendar days from the EMS OHCA incident date. The analysis was carried out pre-linkage and postlinkage, illustrating linkage effects. Thirty-day survival was calculated using OHCAO data where there was a date of death or date discharged $>30$ days after the OHCA incident date. Where there was an OHCAO date of death $\leq 30$ days after the OHCA incident date or further ambulance service data indicating the patient was deceased on the day of the OHCA incident date (eg, hospital code indicating patient deceased and not conveyed to hospital), the patient was categorised as not surviving to 30 days. All other cases were categorised as unknown for patient's 30-day survival status. For the combined linked dataset, cases that were linked to ONS mortality data were categorised as 30-day survival where there was no date of death or where a date of death was provided that was $>30$ days after the OHCA incident date. Where there was an ONS date of death $\leq 30$ days after the OHCA incident date, the patient was categorised as not surviving to 30 days. Where there was a contradiction in patient survival status between OHCAO data and ONS mortality data, then ONS mortality data superseded OHCAO data.

\section{RESULTS}

\section{OHCAO data cleaning process}

Of the 3120 cases transferred to NHS Digital, 2070 $(66.3 \%)$ were automatically matched by the NHS Digital list cleaning algorithm while $1050(33.7 \%)$ were not (figure 1). In total, $620(19.9 \%)$ cases failing automatic matching were resubmitted for manual matching following which 437 (14.0\%) were returned having been manually matched. Also, 430 cases $(13.8 \%)$ were not resubmitted for manual matching as there was little chance of a match due to missing data points (252 cases only had 1 data point out of surname, forename, DOB and home postcode and 178 cases did not have any data points). Overall, $2513(80.5 \%)$ cases were matched of which $7(0.2 \%)$ cases could not be released due to the patient being lost to follow-up (one case, reason unknown) or the patient had registered a type 2 opt-out with NHS Digital, meaning that the patient's personal confidential data could not be released by NHS Digital for reasons other than their own direct care (six cases). In total, $607(19.5 \%)$ cases could not be matched due to insufficient data for matching.

\section{Data points required for matching through NHS Digital list cleaning}

The percentage of each available demographic data point in the random sample of 3120 cases was similar to the 


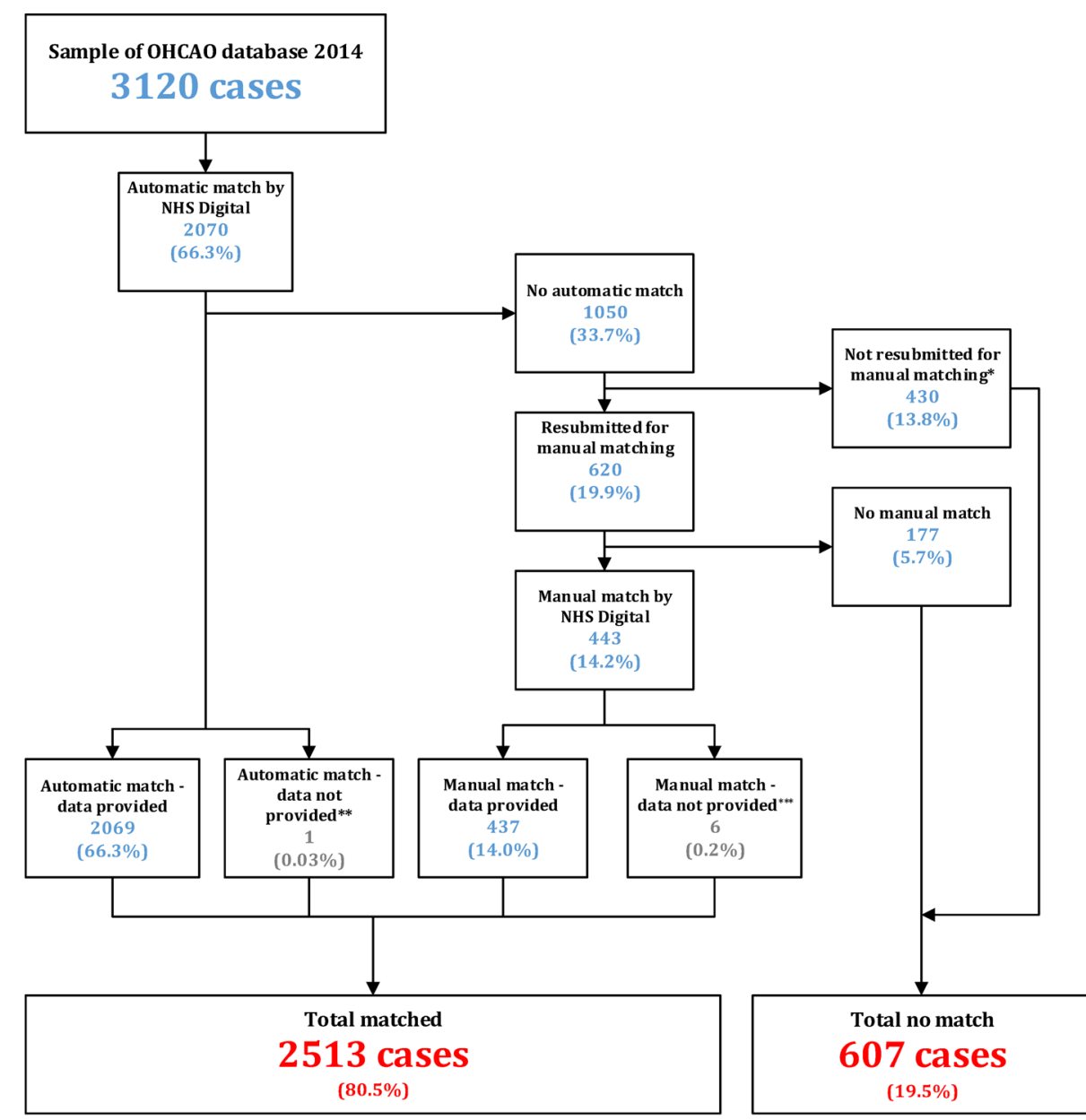

* Cases not resubmitted due to insufficient OHCAO data points to enable NHS Digital to match OHCAO cases to the NHS PDS database.

** Data not provided as patient lost to follow-up (reason unknown).

*** Data not provided due to patient registration of type 2 opt-out with NHS Digital.

Figure 1 Data matching process linking Out-of-Hospital Cardiac Arrest Outcomes (OHCAO) data with National Health Service (NHS) Personal Demographics Service (PDS) data through the NHS Digital list cleaning and patient status service.

percentage of each available demographic data point in all 28729 cases for 2014 (table 1). The data point determining the highest match rate was NHS number. One hundred per cent of cases with an NHS number were matched to the PDS database and therefore matched to ONS mortality data with $99 \%$ of cases with an NHS number being automatically matched. However, only $31.7 \%$ of OHCAO cases had an NHS number.

Approximately a quarter $(27.8 \%)$ of the sample had all 5 data points allowing a match to NHS PDS data (table 2).

Table 1 Total cases with each demographic data point collected by Out-of-Hospital Cardiac Arrest Outcomes (OHCAO) project

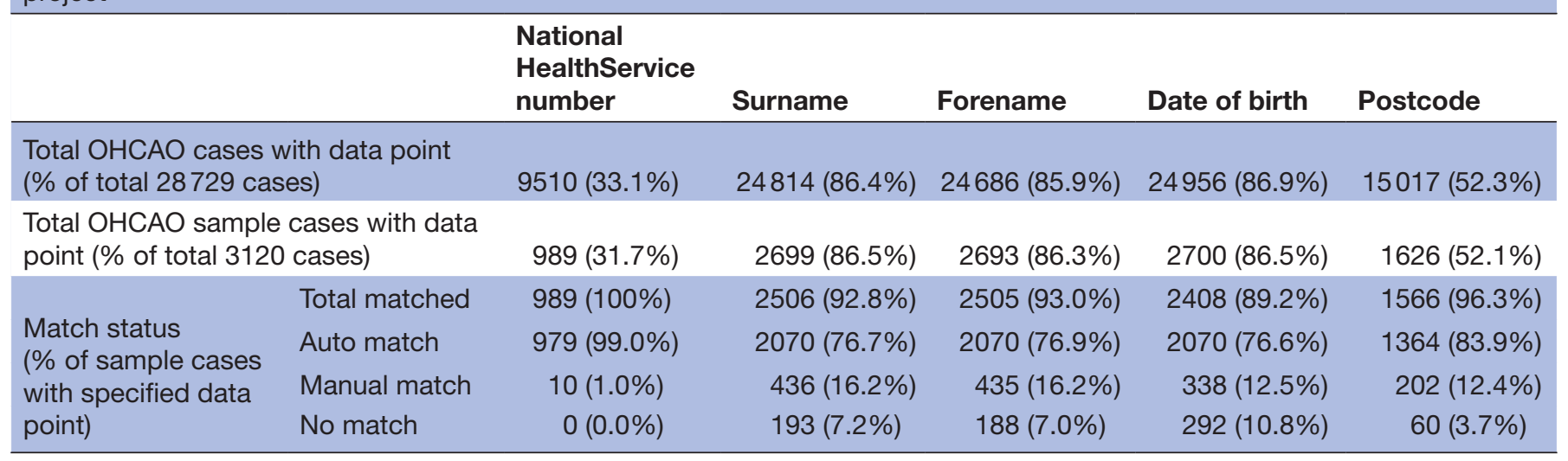


Table 2 Combinations of data points required for linkage to the National Health Service (NHS) Personal Demographics Service database

\section{Data points, $n$ (n cases, \% of total)}

\section{n}

(total 3120 cases)

5 $(868,27.8 \%)$ 4 $(815,26.1 \%)$

\section{NHS+surname+forename+DOB+postcode}

Matched, 2513

(n, \% of total in data point category)

Unmatched, 607

( $n, \%$ of total in data point category)

\begin{tabular}{|c|c|c|c|}
\hline & NHS+surname+forename+postcode & $3(0.4 \%)$ & 0 \\
\hline & $\mathrm{NHS}+$ surname+forename+DOB & $112(13.7 \%)$ & 0 \\
\hline & Surname+forename+DOB+postcode & $665(81.6 \%)$ & $35(4.3 \%)$ \\
\hline & Total & $780(95.7 \%)$ & $35(4.3 \%)$ \\
\hline \multirow{11}{*}{$\begin{array}{l}3 \\
(846,27.1 \%)\end{array}$} & NHS+surname+forename & $1(0.1 \%)$ & 0 \\
\hline & $\mathrm{NHS}+$ surname+DOB & 0 & 0 \\
\hline & NHS+surname+postcode & 0 & 0 \\
\hline & $\mathrm{NHS}+$ forename+DOB & 0 & 0 \\
\hline & $\mathrm{NHS}+$ forename+postcode & 0 & 0 \\
\hline & $\mathrm{NHS}+\mathrm{DOB}+$ postcode & 0 & 0 \\
\hline & Surname+forename+DOB & $760(89.8 \%)$ & $44(5.2 \%)$ \\
\hline & Surname+forename+postcode & $27(3.2 \%)$ & $11(1.3 \%)$ \\
\hline & Surname+DOB+postcode & $1(0.1 \%)$ & $1(0.1 \%)$ \\
\hline & Forename+DOB+postcode & $1(0.1 \%)$ & 0 \\
\hline & Total & $790(93.4 \%)$ & $56(6.6 \%)$ \\
\hline \multirow{11}{*}{$\begin{array}{l}2 \\
(156,5.0 \%)\end{array}$} & NHS+surname & 0 & 0 \\
\hline & NHS+forename & $1(0.6 \%)$ & 0 \\
\hline & $\mathrm{NHS}+\mathrm{DOB}$ & 0 & 0 \\
\hline & NHS+postcode & 0 & 0 \\
\hline & Surname+forename & $67(42.9 \%)$ & $82(52.6 \%)$ \\
\hline & Surname+DOB & 0 & 0 \\
\hline & Surname+postcode & 0 & $2(1.3 \%)$ \\
\hline & Forename+DOB & 0 & $1(0.6 \%)$ \\
\hline & Forename+postcode & 0 & $1(0.6 \%)$ \\
\hline & DOB+postcode & $1(0.6 \%)$ & $1(0.6 \%)$ \\
\hline & Total & $69(44.2 \%)$ & 87 (55.8\%) \\
\hline \multirow{6}{*}{$\begin{array}{l}1 \\
(257,8.2 \%)\end{array}$} & NHS & $4(1.6 \%)$ & 0 \\
\hline & Surname & $2(0.8 \%)$ & $18(7.0 \%)$ \\
\hline & Forename & 0 & $14(5.4 \%)$ \\
\hline & DOB & 0 & 210 (81.7\%) \\
\hline & Postcode & 0 & 9 (3.5\%) \\
\hline & Total & $6(2.3 \%)$ & 251 (97.7\%) \\
\hline $0(178,5.7 \%)$ & $\mathrm{Nil}$ & 0 & $178(100 \%)$ \\
\hline
\end{tabular}

$\mathrm{DOB}$, date of birth.

$53.2 \%$ had 3-4 data points of which $93.4 \%$ and $95.7 \%$ were matched, respectively. Of these, all with NHS numbers were matched, while a combination of data points
surname+forename+DOB+postcode and surname+forename+DOB resulted in match rates of $81.6 \%$ and $89.8 \%$. However, cases where only 1 or 2 data points were 
Table 3 Number of data points added to Out-of-Hospital Cardiac Arrest Outcomes data after NHS Digital list cleaning

\begin{tabular}{lc}
\hline $\begin{array}{l}\text { Number of demographic data points } \\
\text { increased by list cleaning }\end{array}$ & Cases (n) \\
\hline 0 & $1484(47.6 \%)$ \\
\hline 1 & $804(25.8 \%)$ \\
2 & $825(26.4 \%)$ \\
\hline 3 & $7(0.2 \%)$ \\
\hline
\end{tabular}

provided were less likely to be matched $(2.3 \%$ and $44.2 \%$, respectively). In total, $178(5.7 \%)$ cases had no OHCAO demographic data and could not be matched.

Data improvements after NHS Digital list cleaning and provision of ONS date of death data

Demographic improvements

After case matching, NHS Digital returned demographic data (forename, surname, NHS number, home postcode) and ONS date of death if applicable. In total, 1484 (47.6\%) cases were not improved for any demographic data points (table 3). These cases were those where complete demographic data were already collected by OHCAO (868 cases), matching failed (607 cases) or data could not be released by NHS Digital due to the patient either being lost to follow-up or registering a type 2 opt-out with NHS Digital (six of the seven cases). All demographic data were already collected by OHCAO for 1 case out of these 7 cases and therefore were included in the aforementioned 868 cases. Lastly, for three cases OHCAO collected NHS+surname+forename+postcode and therefore these effectively could not be improved by matching as NHS Digital was not asked to provide DOB.

Of the 2249 cases with missing data, $1636(72.7 \%)$ cases had demographic improvements following linkage. A quarter $(25.8 \%)$ were improved by 1 demographic data point and a further quarter (26.4\%) by 2 data points. Of the seven that were improved by 3 data points (table 3 ), OHCAO provided NHS number for four cases, surname for two and DOB and postcode for one.

NHS Digital returned NHS numbers for 1518 (48.7\%) cases in which it was not already collected by OHCAO (see online supplementary table 1). OHCAO had already collected forename and surname in most cases $(86.5 \%$ and $96.3 \%$, respectively) which were least improved following matching.

\section{Survival data improvements following provision of ONS date of death data}

Thirty-day survival status (yes or no) using OHCAO data was confirmed for $1682(53.9 \%)$ cases (table 4$)$. Thirty-day survival was confirmed using OHCAO data if ambulance services provided a date of death or discharge date over 30 days after the OHCA incident date. Linking to ONS mortality data resulted in calculation of 30-day survival status (yes or no) for $2856(91.5 \%)$ cases, a $37.6 \%$ improvement in 30 -day survival status confirmation. The
Table 4 Comparison of 30-day survival calculation predata and post-data linkage

\section{0-day survival}

\begin{tabular}{lclc}
\hline & & \multicolumn{2}{l}{ Dataset 2: linked OHCAO and } \\
Dataset 1: OHCAO data & \multicolumn{2}{l}{ ONS data } \\
\hline Yes & $12(0.4 \%)$ & Yes & $290(9.3 \%)$ \\
No & $1670(53.5 \%)$ & No & $2566(82.2 \%)$ \\
Unknown & $1438(46.1 \%)$ & Unknown & $264(8.5 \%)$ \\
Total & $3120(100 \%)$ & Total & $3120(100 \%)$ \\
\hline
\end{tabular}

OHCAO, Out-of-Hospital Cardiac Arrest Outcomes; ONS, Office for National Statistics.

pre-linkage 30-day survival rate was calculated as $0.4 \%$ and post-linkage as $9.3 \%$.

\section{Accuracy of OHCAO date of death data}

In this sample, OHCAO reported a date of death for 1178 $(37.8 \%)$ cases from ambulance services. In seven $(0.6 \%)$ cases, death was not recorded with the ONS at the time of linkage. Of the $1942(62.2 \%)$ cases where OHCAO could not confirm a date of death, $248(12.8 \%)$ were recorded as alive at the time of linkage and $1137(58.5 \%)$ had died according to ONS mortality data (table 5 ).

\section{DISCUSSION}

This study demonstrates the feasibility of linking OHCAO data to NHS patient demographic data and ONS date of death data through NHS Digital. In this sample of 3120 OHCAO cases, an $80.5 \%$ match rate was achieved and this enabled provision of registered death dates to calculate 30-day survival status. The results showed a 30-day survival rate of $9.3 \%$, reducing unknown survival status from $46.1 \%$ to $8.5 \%$ (table 4). Additionally, demographic data quality improved for 1636 (52.4\%) cases, with NHS numbers being provided for $1518(48.7 \%)$ cases and postcodes for $942(30.2 \%)$ cases where these data were missing in the OHCAO database.

Table 5 Comparison of date of death confirmed by Out-ofHospital Cardiac Arrest Outcomes (OHCAO) and Office for National Statistics (ONS) data

\begin{tabular}{|c|c|c|c|c|c|}
\hline & & \multicolumn{3}{|c|}{$\begin{array}{l}\text { ONS confirmed survival } \\
\text { status }\end{array}$} & \multirow{2}{*}{$\begin{array}{l}\text { Total }(n, \% \\
\text { total } 3120 \\
\text { cases) }\end{array}$} \\
\hline & & Dead & Alive & No & \\
\hline \multirow{2}{*}{$\begin{array}{l}\text { OHCAO } \\
\text { project } \\
\text { date of } \\
\text { death } \\
\text { provided }\end{array}$} & Yes & 1114 & 7 & 57 & $\begin{array}{l}1178 \\
(37.8 \%)\end{array}$ \\
\hline & No & 1137 & 248 & 557 & $\begin{array}{l}1942 \\
(62.2 \%)\end{array}$ \\
\hline \multicolumn{2}{|c|}{$\begin{array}{l}\text { Total ( } n, \% \text { total } \\
3120 \text { cases) }\end{array}$} & $\begin{array}{l}2251 \\
(72.1 \%)\end{array}$ & $\begin{array}{l}255 \\
(8.2 \%)\end{array}$ & $\begin{array}{l}614^{*} \\
(19.7 \%)\end{array}$ & $\begin{array}{l}3120 \\
(100 \%)\end{array}$ \\
\hline
\end{tabular}

*Includes seven matched cases where data not provided by NHS Digital (one patient lost to follow-up (reason unknown), six patient registration of type 2 opt-out with NHS Digital). 
The variability of cardiac arrest survival across ambulance services in England has been previously highlighted. ${ }^{6}$ Where data from ambulance services do not follow a standard procedure, data collection variability may have significant effects on data quality and comparability between services. Increasingly, core outcome sets for specific research areas are developed outlining minimum datasets for routine collection and create a level of standardisation to compare studies and allow formation of meta-analyses. ${ }^{30}$ In the field of OHCA, the Utstein guidelines have been developed. ${ }^{16}{ }^{17}$ However, a study investigating the level of missing data within primary outcomes in 283 Cochrane Reviews of all areas of clinical practice found that $>50 \%$ of patient data were missing in $18 \%$ of reviews. ${ }^{31}$ Furthermore, an analysis of 12 international OHCA registries collection of data using Utstein templates found that although all registries collected core variables, there were differences in interpretation of the template and recorded 'unknown' for a mean of 4.8 variables and 'missing' for 1.9 variables. ${ }^{32}$ Therefore, minimum datasets are not sufficiently effective in reducing missing data.

The best data point provided by ambulance services to identify cases in the UK is the NHS number. It provides a unique identifier to resolve missing demographic data issues if no other demographic data are provided. One hundred per cent of OHCAO cases with an NHS number were matched to NHS PDS data; however, it was only available in a third (31.7\%) of cases (table 1). Logistical difficulties exist in ascertaining an NHS number as it may not be available in the out-of-hospital setting. However, this study found that providing at least 3-4 demographic variables other than an NHS number resulted in a match rate of up to $89.8 \%$, depending on the combination and especially if forename and surname were provided. This also allowed provision of an NHS number in $48.7 \%$ of cases where it was not collected by the OHCAO project (see online supplementary table 1 ). If less than a threshold of 3 data points were provided, this study found a lower potential for matching $(0 \%-44.2 \%$, table 2$)$. Our findings support previous research showing that the ability to successfully link international OHCA databases to outcome data is dependent on the provision and completeness of patient identifiers. For example, the Danish Cardiac Arrest Registry was able to link to the Danish Civil Registration System to confirm 30-day survival for $100 \%$ of OHCA patients due to $100 \%$ provision of a unique Civil Registration Number. ${ }^{20}$ Conversely, a study from the USA showed limited feasibility for linking OHCA patients to longitudinal outcomes when there was no unique patient identifiers available and there was variability in completeness of patient demographic data, resulting in a linkage rate of only $34.2 \% .^{33}$

NHS Digital list cleaning increased the number of OHCAO cases with a validated NHS number by 1518 $(48.7 \%)$ to $2507(80.4 \%)$ cases, suggesting that data linkage is a feasible method for linking an OHCA dataset to the national mortality dataset. The current process for ambulance services in England to confirm survival to discharge from OHCA is challenging, ${ }^{11} 12$ and using the NHS Digital list cleaning and patient status service to calculate 30-day survival from OHCA may be a viable alternative. The results of this study also suggest the potential to use data linkage for further avenues of research relating to OHCA in the UK. Data linkage can be used to follow OHCA patients longitudinally, for example, to investigate predictors of survival at 1 year, 5 years and beyond. ${ }^{44}$ Furthermore, data linkage can be used to evaluate the complete patient care pathway by linking to existing routinely collected hospital data sources. For example, hospital interventions and hospital length of stay via Hospital Episode Statistics and intensive care interventions via the Intensive Care National Audit and Research Centre. NHS Digital also provided postcodes for a further 942 (30.2\%) cases, which increases the potential to examine the influence of neighbourhood characteristics, such as population density and social deprivation, on OHCA incidence, whether an event is witnessed, and if they receive bystander cardiopulmonary resuscitation (CPR). ${ }^{35} 36$

The OHCAO project was able to collect a date of death for $1178(37.8 \%)$ cases. Interestingly, a date of death was not recorded with the ONS for seven of these cases, indicating that the patients were still alive at the time of linkage. Such errors may lead to incorrect reporting of cardiac arrest survival as part of the NHS England AQIs. This is an important finding as this shows the importance of data linkage to correct database errors.

This study's strengths lie in its standardised procedures for OHCA case definition and data collection, with the data points collected based on established Utstein guidelines. ${ }^{16}$ A further strength is that NHS Digital used both deterministic and probabilistic data linkage methods; they have different strengths and using both methods may enhance linkage performance. ${ }^{37}$ Deterministic linkage methods have greater specificity but require exact matches between records, while probabilistic data linkage has greater sensitivity, working better with poorer quality data as it allows imperfect matches between records. ${ }^{27}$ For example, the returned demographic data for the linked cases showed that 14 OHCAO cases with between 4 and 5 data points were linked despite having an erroneous NHS number. This allowed correction of the inaccurate NHS number in the OHCAO sample. Finally, successful data linkage enabled access to high-quality national date of death data from ONS that is subject to rigorous data quality and validation processes. ${ }^{28}$

\section{Limitations}

This study had several limitations. First, only 868 (27.8\%) cases had all 5 OHCAO data points, while $178(5.7 \%)$ cases had missing data for all OHCAO data points. Missing data is an issue in OHCA registries, ${ }^{32}$ and improved data linkage in the OHCAO project is reliant on improved data capture of patient demographic data by ambulance services. While NHS numbers were provided for only 989 $(31.7 \%)$ OHCAO cases, one ambulance service provided 
NHS numbers for $100 \%$ of their cases. This suggests potential for the OHCAO project to work with ambulance services to increase provision of patient demographic data to improve data linkage. Second, following linkage 30-day survival status remained unknown for $264(8.5 \%)$ cases. Data not missing completely at random can bias results. ${ }^{38}$ For example, if those 264 patients survived to 30 days the overall 30-day survival rate would be $17.8 \%$ (584 cases) instead of $9.3 \%$ (290 cases). Third, where no date of death was provided, cases were categorised as alive. However, absence of recorded death may mean registration of death has been delayed, for example, due to a coroner's inquest. Although it should also be noted that NHS Digital did not commence data linkage until $>12$ months (March 2016) after the date (31 January 2015) where 30-day survival could be calculated for patients in the sample suffering an OHCA on 31 December 2014. ONS data for 2014-2015 show that only $6.1 \%$ of deaths in England and Wales required a coroner's inquest ${ }^{28}$ and the average time of an inquest was 24 weeks. ${ }^{39} 40$ Furthermore, ONS data from 2011 report that overall $94 \%$ of deaths were registered within 1 month. ${ }^{29}$ Finally, where the quality and completeness of data is variable data linkage errors can occur, which can bias reported outcomes. ${ }^{41}$ Deterministic data linkage methods increase the likelihood of false negative matches (not matching to a correct match), while probabilistic data linkage increases the likelihood of false positive matches (matching to an incorrect match). ${ }^{27}$ To quantify how data linkage errors may impact on study findings and outcomes, a formal data linkage validation evaluation is required. ${ }^{18}$ This was beyond the scope of this study but should be conducted if OHCAO establishes a data linkage programme.

\section{CONCLUSIONS}

This study shows the feasibility of linking data from the UK OHCAO project to NHS patient demographic and ONS date of death data using the NHS Digital list cleaning and patient status service. This enabled analysis of 30-day survival status which may be of use to the NHS in terms of resource planning and directing service provision. Missing NHS numbers are a significant obstacle to successful data linkage, and this study found that if at least forename and surname are collected with one other demographic data point, there is a high chance of retrieving missing NHS numbers. Demographic data were improved for over half of cases and can be used as a means of creating a registry of OHCA patients to investigate postresuscitation care and longitudinal outcomes.

Acknowledgements National Ambulance Services Clinical Quality Group and the National Ambulance Research Steering Group.

Collaborators Dr Sukhdeep Dosanjh, Warwick Clinical Trials Unit, University of Warwick; Theresa Foster, East of England Ambulance Service NHS Trust; Frank Mersom, East of England Ambulance Service NHS Trust; Gurkamal Francis, London Ambulance Service NHS Trust; Michelle 0'Rourke, North East Ambulance Service NHS Trust; Clare Bradley, North West Ambulance Service NHS Trust; Philip King, South Central Ambulance Service NHS Trust; Ed England, South Central Ambulance
Service NHS Trust; Patricia Bucher, South East Coast Ambulance Service NHS Trust; Jessica Lynde, South Western Ambulance Service NHS Trust; Nancy Loughlin, South Western Ambulance Service NHS Trust; Jenny Lumley-Holmes, West Midlands Ambulance Service NHS Trust; Dr Julian Mark, Yorkshire Ambulance Service NHS Trust.

Contributors GDP designed the study. SJB, CJ, CH, ANS, KK, SB, RS and IG contributed to data collection. SR analysed the data and wrote the initial draft of the paper. SR, GDP and SJB were involved in further drafting of the paper. All authors participated in interpreting the data, revising the paper for critically important intellectual content and gave final approval of the submitted version.

Funding This work was supported by research grants from the British Heart Foundation and Resuscitation Council (UK).

Competing interests SJB, TPB, CJ, CH, SJBM and GDP are employed by the University of Warwick, which receives grants from the British Heart Foundation and the Resuscitation Council (UK) for the conduct of the OHCAO project.

Ethics approval Ethical approval for the OHCAO project was gained from the National Research Ethics Committee South Central, reference number 13/SC/0361. Confidential Advisory Group (CAG) approval was granted, reference number ECC 8-04(c)/2013, to collect identifiable patient information where it is not practical to obtain consent.

Provenance and peer review Not commissioned; externally peer reviewed.

Data sharing statement Please refer to the OHCAO project website for information relating to data sharing requests: http://www2.warwick.ac.uk/fac/med/ research/hscience/ctu/trials/ohcao/health/data/data_sharing/

Open Access This is an Open Access article distributed in accordance with the Creative Commons Attribution Non Commercial (CC BY-NC 4.0) license, which permits others to distribute, remix, adapt, build upon this work non-commercially, and license their derivative works on different terms, provided the original work is properly cited and the use is non-commercial. See: http://creativecommons.org/ licenses/by-nc/4.0/

(c) Article author(s) (or their employer(s) unless otherwise stated in the text of the article) 2017. All rights reserved. No commercial use is permitted unless otherwise expressly granted.

\section{REFERENCES}

1. Resuscitation Council (UK), NHS England, British Heart Foundation. Consensus paper on out-of-hospital cardiac arrest in England: Resuscitation Council, 2014.

2. Ambulance Service Association. National cardiac arrest audit report. London: Ambulance Service Association, 2006.

3. Sasson C, Rogers MA, Dahl J, et al. Predictors of survival from outof-hospital cardiac arrest: a systematic review and meta-analysis. Circ Cardiovasc Qual Outcomes 2010;3:63-81.

4. Shuvy M, Morrison LJ, Koh M, et al. Long-term clinical outcomes and predictors for survivors of out-of-hospital cardiac arrest. Resuscitation 2017;112:59-64.

5. Institute of Medicine Committee on the Treatment of Cardiac Arrest. Strategies to improve cardiac arrest survival. A time to act: National Academies Press, 2015.

6. Perkins GD, Cooke MW. Variability in cardiac arrest survival: the NHS ambulance service quality indicators. Emerg Med J 2012;29:3-5.

7. Girotra S, van Diepen S, Nallamothu BK, et al. Regional variation in out-of-hospital cardiac arrest survival in the United States. Circulation 2016;133:2159-68.

8. Okubo M, Kiyohara K, Iwami T, et al. Nationwide and regional trends in survival from out-of-hospital cardiac arrest in Japan: A 10-year cohort study from 2005 to 2014. Resuscitation 2017;115:120-8.

9. Strömsöe A, Svensson L, Axelsson ÅB, et al. Improved outcome in Sweden after out-of-hospital cardiac arrest and possible association with improvements in every link in the chain of survival. Eur Heart $J$ 2015;36:863-71.

10. Lilford R, Mohammed MA, Spiegelhalter D, et al. Use and misuse of process and outcome data in managing performance of acute medical care: avoiding institutional stigma. Lancet 2004;363:1147-54.

11. Fothergill R, Brace-McDonnell SJ, Perkins GD. Variation in epidemiology and outcomes from cardiac arrest. Resuscitation 2014;85:1610-1.

12. Perkins GD, Lall R, Quinn T, et al. Mechanical versus manual chest compression for out-of-hospital cardiac arrest (PARAMEDIC): 
a pragmatic, cluster randomised controlled trial. Lancet 2015;385:947-55.

13. Berdowski J, Berg RA, Tijssen JG, et al. Global incidences of outof-hospital cardiac arrest and survival rates: Systematic review of 67 prospective studies. Resuscitation 2010;81:1479-87.

14. Ong ME, Shin SD, De Souza NN, et al. Outcomes for out-ofhospital cardiac arrests across 7 countries in Asia: The Pan Asian Resuscitation Outcomes Study (PAROS). Resuscitation 2015;96:100-8.

15. Gräsner JT, Lefering R, Koster RW, et al. EuReCa ONE-27 Nations, ONE Europe, ONE Registry: a prospective one month analysis of out-of-hospital cardiac arrest outcomes in 27 countries in Europe. Resuscitation 2016;105:188-95.

16. Jacobs I, Nadkarni V, Bahr J, et al. Cardiac arrest and cardiopulmonary resuscitation outcome reports: update and simplification of the Utstein templates for resuscitation registries. Resuscitation 2004;63:233-49.

17. Perkins GD, Jacobs IG, Nadkarni VM, et al. Cardiac arrest and cardiopulmonary resuscitation outcome reports: update of the utstein resuscitation registry templates for out-of-hospital cardiac arrest. Resuscitation 2015;96:328-40.

18. Moore CL, Amin J, Gidding HF, et al. A new method for assessing how sensitivity and specificity of linkage studies affects estimation. PLoS One 2014;9:e103690-8.

19. Elmer J, Rittenberger JC, Coppler PJ, et al. Long-term survival benefit from treatment at a specialty center after cardiac arrest. Resuscitation 2016;108:48-53.

20. Hamilton A, Steinmetz J, Wissenberg M, et al. Association between prehospital physician involvement and survival after out-ofhospital cardiac arrest: A Danish nationwide observational study. Resuscitation 2016:108:95-101.

21. Perkins GD, Brace-McDonnell SJ. The UK Out of Hospital Cardiac Arrest Outcome (OHCAO) project. BMJ Open 2015;5:e008736.

22. Hawkes $\mathrm{C}$, Booth $\mathrm{S}$, Ji C, et al. Epidemiology and outcomes from out-of-hospital cardiac arrests in England. Resuscitation 2017;110:133-40.

23. Office for National Statistics Annual Mid-Year Population Estimates. 2014 http://www.ons.gov.uk/peoplepopulationandc ommunity/populationandmigration/populationestimates/bulletins/ annualmidyearpopulationestimates/2015-06-25(Accessed 7 July 2017).

24. Joint Royal Colleges Ambulance Liaison Committee and Association of Ambulance Chief Executives. UK Ambulance Services Clinical Practice Guidelines 2016. Bridgwater: Class Professional Publishing, 2016.

25. Jaro MA. Probabilistic linkage of large public health data files. Stat Med 1995;14:491-8.

26. Méray N, Reitsma JB, Ravelli AC, et al. Probabilistic record linkage is a valid and transparent tool to combine databases without a patient identification number. J Clin Epidemiol 2007;60:883.e1-883.e11.
27. Zhu Y, Matsuyama Y, Ohashi Y, et al. When to conduct probabilistic linkage vs. deterministic linkage? A simulation study. J Biomed Inform 2015;56:80-6.

28. Office for National Statistics User guide to mortality statistics. 2017 https://www.ons.gov.uk/peoplepopulationandcommunity/birthsde athsandmarriages/deaths/methodologies/userguidetomortalitys tatisticsjuly2017

29. Office for National Statistics Impact of registration delays on mortality statistics. $2011 \mathrm{http}: / /$ webarchive.nationalarchives.gov.uk/ 20160106020016/http://www.ons.gov.uk/ons/guide-method/userguidance/health-and-life-events/impact-of-registration-delays-onmortality-statistics/index.html

30. Kirkham JJ, Gorst S, Altman DG, et al. Core Outcome SetSTAndards for Reporting: The COS-STAR Statement. PLOS Med 2016:13:e1002148.

31. Kirkham JJ, Gargon E, Clarke M, et al. Can a core outcome set improve the quality of systematic reviews?--a survey of the Coordinating Editors of Cochrane Review Groups. Trials 2013;14:21.

32. Nishiyama C, Brown SP, May S, et al. Apples to apples or apples to oranges? International variation in reporting of process and outcome of care for out-of-hospital cardiac arrest. Resuscitation 2014;85:1599-609.

33. Mumma BE, Diercks DB, Danielsen B, et al. Probabilistic Linkage of Prehospital and Outcomes Data in Out-of-hospital Cardiac Arrest. Prehosp Emerg Care 2015;19:358-64.

34. Dumas F, Rea TD. Long-term prognosis following resuscitation from out-of-hospital cardiac arrest: role of aetiology and presenting arrest rhythm. Resuscitation 2012;83:1001-5.

35. Fosbøl EL, Dupre ME, Strauss B, et al. Association of neighborhood characteristics with incidence of out-of-hospital cardiac arrest and rates of bystander-initiated CPR: implications for community-based education intervention. Resuscitation 2014;85:1512-7.

36. Moon S, Bobrow BJ, Vadeboncoeur TF, et al. Disparities in bystander CPR provision and survival from out-of-hospital cardiac arrest according to neighborhood ethnicity. Am J Emerg Med 2014;32:1041-5.

37. Gomatam S, Carter R, Ariet M, et al. An empirical comparison of record linkage procedures. Stat Med 2002;21:1485-96.

38. He Y. Missing data analysis using multiple imputation. Circ Cardiovasc Qual Outcomes 2010;3:98-105.

39. Ministry of Justice Coroners Statistics 2014 England and Wales. 2015 https://www.gov.uk/government/uploads/system/uploads/ attachment_data/file/427720/coroners-statistics-2014.pdf

40. Ministry of Justice Coroners Statistics 2015 England and Wales. 2016 https://www.gov.uk/government/uploads/system/uploads/ attachment_data/file/607728/coroners-statistics-2015.pdf

41. Hagger-Johnson G, Harron K, Fleming T, et al. Data linkage errors in hospital administrative data when applying a pseudonymisation algorithm to paediatric intensive care records. BMJ Open 2015:5:e008118. 